\title{
Establishing a Federal and State Data Exchange Pilot for Public Health Situational Awareness
}

\author{
Dina B. Passman*1, Aaron Kite-Powell², Dara Spector ${ }^{1}$, Wayne Loschen ${ }^{3}$, Barry Harp ${ }^{1}$, \\ Aaron Chern², Janet Hamilton ${ }^{2}$, Cary Eggers ${ }^{2}$ and Joseph Lombardo 3
}

1U.S. Department of Health and Human Services, Office of the Assistant Secretary for Preparedness and Response, Washington, DC, USA; ' Florida Department of Health, Bureau of Epidemiology, Jacksonville, FL, USA; ${ }^{3}$ Johns Hopkins University Applied Physics Laboratory, Laurel, MD, USA

\section{Objective}

U.S. Department of Health and Human Services (HHS) Office of the Assistant Secretary for Preparedness and Response (ASPR) partnered with the Florida Department of Health (FDOH), Bureau of Epidemiology, to implement a new process for the unidirectional exchange of electronic medical record (EMR) data when ASPR clinical assets are operational in the state following a disaster or other response event. The purpose of the current work was to automate the exchange of data from the ASPR electronic medical record system EMR-S into the FDOH Electronic Surveillance System for the Early Notification of Community-based Epidemics (ESSENCE-FL) system during the 2012 Republican National Convention (RNC).

\section{Introduction}

ASPR deploys clinical assets, including an EMR system, to the ground per state requests during planned and no-notice events. The analysis of patient data collected by deployed federal personnel is an integral part of ASPR and FDOH's surveillance efforts. However, this surveillance can be hampered by the logistical issues of field work in a post-disaster environment leading to delayed analysis and interpretation of these data to inform decision makers at the federal, state, and local levels. FDOH operates ESSENCE-FL, a multi-tiered, automated, and secure web-based application for analysis and visualization of clinical data. The system is accessible statewide by FDOH staff as well as by hospitals that participate in the system. To improve surveillance ASPR and FDOH engaged in a pilot project whereby EMR data from ASPR would be sent to FDOH in near realtime during the 2012 hurricane season and the $2012 \mathrm{RNC}$. This project is in direct support of Healthcare Preparedness Capability 6, Information Sharing, and Public Health Preparedness Capability 13, Public Health Surveillance and Epidemiological Investigation.

\section{Methods}

In 2011, FDOH approached ASPR about securely transmitting raw EMR data that could be ingested by ESSENCE-FL during ASPR deployments in the state. Upon conclusion of an agreement for a date exchange pilot, data elements of interest from the ASPR EMR were identified. Due to the modular design ESSENCE-FL Microsoft SQL databases were easily adapted by the Johns Hopkins University Applied Physics Laboratory (JHU/APL) to add a new module to handle receipt of ASPR EMR data including code to process the files, remove duplicates and create associations with existing reference in- formation, such as system-defined geographic regions and age groups. Scripts were developed to run on the ASPR server to create and send updated files via secure file transfer protocol (SFTP) every 15 minutes to ESSENCE-FL. Prior ASPR event deployment data was scrubbed and sent to ESSENCE-FL as a test dataset to ensure appropriate receipt and ingestion of the new data source.

\section{Results}

EMR data was transmitted through a central server at ASPR to ESSENCE-FL every 15 minutes during each day of the 2012 RNC (August 26-31). In ESSENCE-FL, configuration allowed the data to be queried, analyzed, and visualized similar to existing ESSENCEFL data sources. In all, data from 11 patient encounters were successfully exchanged between the partners. The data were used by ASPR and FDOH to simultaneously monitor in near real-time onsite medical response activities during the convention.

\section{Conclusions}

Timely access to patient data can enhance situational awareness and disease surveillance efforts and provide decision makers with key information in an expedient manner during disaster response and mass gatherings such as the RNC. However, data are siloed within organizations. The collaboration between FDOH, ASPR and JHU/APL made EMR data sharing and analysis more expeditious and efficient and increased timely access to these data by local, state, and federal epidemiologists. The integration of these data into the ESSENCE-FL system created one location where users could go to access data and create epidemiologic reports for a given region in Florida, including the RNC. To achieve these successes with partners in the future, it will be necessary to develop partnerships well in advance of intended data exchange. Future recommendations include robust pre-event testing of the data exchange process and planning for a greater amount of lead-time between enacting the official agreement and beginning data exchange.

\section{Keywords}

Syndromic surveillance; Public health informatics; Data exchange; Federal and state collaboration

*Dina B. Passman

E-mail: dina.passman@hhs.gov 